
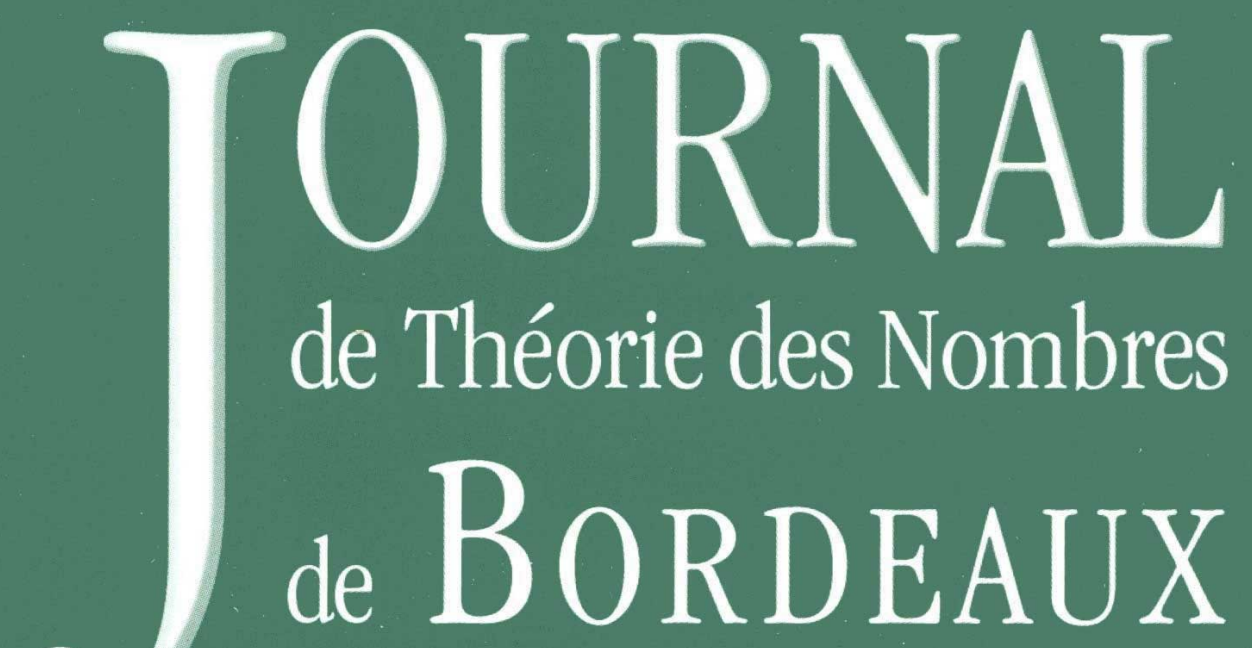

anciennement Séminaire de Théorie des Nombres de Bordeaux

\title{
Francesco BATTISTONI
}

\section{On small discriminants of number fields of degree 8 and 9}

Tome 32, nº 2 (2020), p. 489-501.

<http://jtnb.centre-mersenne.org/item?id=JTNB_2020__32_2_489_0>

(C) Société Arithmétique de Bordeaux, 2020, tous droits réservés. L'accès aux articles de la revue «Journal de Théorie des Nombres de Bordeaux » (http://jtnb.centre-mersenne.org/), implique l'accord avec les conditions générales d'utilisation (http://jtnb. centre-mersenne.org/legal/). Toute reproduction en tout ou partie de cet article sous quelque forme que ce soit pour tout usage autre que l'utilisation à fin strictement personnelle du copiste est constitutive d'une infraction pénale. Toute copie ou impression de ce fichier doit contenir la présente mention de copyright.

\section{cedram}

Article mis en ligne dans le cadre du

Centre de diffusion des revues académiques de mathématiques

http://www.centre-mersenne.org/ 


\title{
On small discriminants of number fields of degree 8 and 9
}

\author{
par FRANCESCO BATTISTONI
}

\begin{abstract}
RÉSumÉ. Nous classifions tous les corps de nombres de signature $(4,2),(6,1)$, $(1,4)$ et $(3,3)$ et discriminant inférieur à une certaine borne spécifique. Ceci achève la recherche du discriminant minimal pour les corps de degré 8 et contribue à l'étude du cas de degré 9 . On rappelle les outils théoriques et les étapes algorithmiques sur lesquels repose notre méthode, on se concentre ensuite sur les aspects nouveaux qui proviennent de la nouvelle implémentation de ce processus dans le système de calcul formel PARI/GP; enfin, on fait quelques remarques sur nos résultats finals, parmi lesquels mentionnons l'existence d'un corps de nombres de signature $(3,3)$ et d'un petit discriminant, inconnu jusqu'à présent.
\end{abstract}

ABSTRACT. We classify all the number fields with signature $(4,2),(6,1),(1,4)$ and $(3,3)$ having discriminant lower than a specific upper bound. This completes the search for minimum discriminants for fields of degree 8 and continues it in the degree 9 case. We recall the theoretical tools and the algorithmic steps upon which our procedure is based, then we focus on the novelties due to a new implementation of this process on the computer algebra system PARI/GP; finally, we make some remarks about the final results, among which the existence of a number field with signature $(3,3)$ and small discriminant which was not previously known.

\section{Introduction}

Consider the family of number fields $K$ with fixed degree $n$ and fixed signature $\left(r_{1}, r_{2}\right)$. Classical results by Minkowski and Hermite, obtained at the end of the 19th century, imply the following properties for the discriminant $d_{K}$ of the fields:

- There exists an explicit lower bound $\left|d_{K}\right|>C\left(n, r_{2}\right)$, where $C\left(n, r_{2}\right)>1$ depends only on the degree and the signature. This implies that in the family of fields with degree $n$ and signature $\left(r_{1}, r_{2}\right)$ there exists a field $F$ such that $\left|d_{K}\right|$ attains the minimum value when $K=F$. 
- For every $C>0$, there exist only finitely many number fields with fixed degree and signature and such that $\left|d_{K}\right| \leq C$.

The study of number fields with respect to their discriminants is then characterized by a double purpose: to find the minimum values for the discriminants of fields with fixed signature, and to completely classify all the fields in this family up to a chosen discriminant bound (a goal which encompasses the first one). Complete tables of number fields with bounded discriminant are useful tools in Number Theory, because they provide explicit examples over which one can get some heuristic or prove results which are known to be asymptotically true in the discriminant (see, as an example, the work by Astudillo, Diaz y Diaz and Friedman [1] on minimum regulators which explicitly requires this kind of lists).

Giving a complete classification of fields with fixed degree $n$ and signature $\left(r_{1}, r_{2}\right)$ is easy for $n=2$, because every quadratic field has the form $\mathbb{Q}(\sqrt{d})$ with $d \in \mathbb{Z}$ squarefree, and this structure returns $d_{K}$ equal to either $4 d$ or $d$, depending on the residue class of $d$ modulo 4 .

For $n=3$ the research is still not difficult, thanks to Davenport and Heilbronn's correspondence between isomorphism classes of cubic orders in number fields and equivalence classes of binary integral cubic forms [9]; this bijection preserves discriminants and is the theoretical cornerstone for Belabas' algorithm for the classification of cubic fields with bounded discriminant [4].

Whenever one considers fields of higher degree, the classification becomes harder to get. There are two main mathematical frameworks, developed during the 1970's and 1980's, which allowed several researchers to get results for fields with low degree:

- Geometry of Numbers and its applications to the rings of integers, which provided explicit estimates on the possible maximum values for the coefficients of the defining polynomials of number fields $K$ with bounded $\left|d_{K}\right|$ : this was investigated by Hunter and Pohst [28] for number fields over $\mathbb{Q}$ and by Martinet [19] for generic number field extensions;

- Lower bounds for the discriminants derived from the explicit formulae of Dedekind Zeta functions, a procedure which was pursued by Odlyzko [20], Poitou [30] and Serre [34] and which allowed Diaz y Diaz [10] to obtain lower bounds of $\left|d_{K}\right|$ for several degrees and signatures.

The simultaneous use of the previous tools permitted to develop algorithmic procedures which gave complete classifications of number fields up to certain discriminant bounds in the following cases:

- Number fields with degree 4 [6] and 5 [31]; 
- Number fields with degree $6[5,21,22,23,24,25,26]$ and degree $7[11,12,14,27]$.

- Totally complex [13] and totally real [29] number fields of degree 8;

- Totally real number fields of degree $9[36]$.

For what concerns further signatures in degree 8 , no complete tables up to some bound were known and for several years no attempts of this kind were made. During his Ph.D. work, the author [3] was then able to give a complete classification of number fields with degree 8 , signature $(2,3)$ and $\left|d_{K}\right| \leq 5726301$, showing that there exist exactly 56 such fields: this result was obtained by exploiting the aforementioned theoretical ideas in order to write an algorithmic procedure which was implemented in a program relying on the softwares MATLAB and PARI/GP [38].

This setting was not good enough for exploring other signatures in degree 8 and 9 , but we are now able to provide a better implementation, needing just PARI/GP, which allowed us to obtain the following classification result.

Theorem 1.1. There exist 41 number fields $K$ with signature $(4,2)$ and with $\left|d_{K}\right| \leq 20829049$. The minimum value of $\left|d_{K}\right|$ is 15243125 .

There exist 8 number fields $K$ with signature $(6,1)$ and with $\left|d_{K}\right| \leq$ 79259702. The minimum value of $\left|d_{K}\right|$ is 65106259 .

There exist 67 number fields $K$ with signature $(1,4)$ and with $\left|d_{K}\right| \leq$ 39657561. The minimum value of $\left|d_{K}\right|$ is 29510281.

There exist 116 number fields $K$ with signature $(3,3)$ and with $\left|d_{K}\right| \leq$ 146723910. The minimum value of $\left|d_{K}\right|$ is 109880167.

The number fields and the complete tables are gathered in the website [2], together with the PARI/GP programs used for their classification, and these programs can be found also in the GitHub repository available at https: // github.com/FrancescoBattistoni/SmallDiscriminants. The programs were run on the cluster system of Université de Bordeaux and on the clusters INDACO and HORIZON of Università degli Studi di Milano.

Here is an overview of the paper.

Section 2 recalls the theoretical foundations of the algorithmic procedure, which are respectively Hunter-Pohst-Martinet's Theorem and the local corrections for lower bounds of discriminants given by prime ideals. Section 3 presents the various steps in which the algorithm is divided. Section 4 finally presents the main novelties of our new implementation and some remarks on the final results obtained.

Acknowledgements. I would like to thank my Ph.D. advisor Giuseppe Molteni for every useful suggestion and for his supervision of my research in Università degli Studi di Milano. I would like to thank also Institut de Mathématiques de Bordeaux, for hosting me and allowing me to use the 
IMB cluster, and the people I worked with and gave me many advices: Bill Allombert, Karim Belabas, Henri Cohen, Andreas Enge, Aurel Page, Guillaume Ricotta, Damien Robert.

Thanks also to Alessio Alessi and Francesco Fichera, who gave me permission for using INDACO and HORIZON clusters respectively, to Schehrazad Selmane, for lending me her tables with local corrections, and to Gunter Malle and Ken Yamamura, for their remarks about the preliminary version of this paper.

\section{Theoretical recalls}

In our procedure we look for irreducible monic polynomials of degree 8 and 9 with integer coefficients which define the desired number fields: the first problem consists then in giving an upper bound to the number of these polynomials, and the bound should depend on the discriminant and the signature.

Given a number field $K$, an element $\alpha \in \mathcal{O}_{K}$ and a number $k \in \mathbb{Z}$, the Newton sum of order $k$ of $\alpha$ is defined as the sum

$$
S_{k}(\alpha):=\sum_{i=1}^{n} \alpha_{i}^{k}
$$

where the $\alpha_{i}$ 's represent the conjugates of $\alpha$ with respects to the embeddings $\sigma_{1}, \ldots, \sigma_{n}$ of $K$.

One has $S_{k}(\alpha) \in \mathbb{Z}$, and $S_{1}(\alpha)=\operatorname{Tr}(\alpha)$; moreover, if $f(x):=x^{n}+$ $a_{1} x^{n-1}+\cdots+a_{n-1} x+a_{n}$ is the defining polynomial of $\alpha$, then one has the recursive relations

$$
S_{k}(\alpha)=-k a_{k}-\sum_{j=1}^{k-1} a_{j} S_{k-j}(\alpha) \quad \text { for every } 2 \leq k \leq n
$$

which link the coefficients of $f(x)$ to the values of the Newton sums.

Consider then the absolute Newton sum

$$
T_{2}(\alpha):=\sum_{i=1}^{n}\left|\alpha_{i}\right|^{2}
$$

We have an estimate for $T_{2}(\alpha)$, depending on $n$ and $\left|d_{K}\right|$, provided by Hunter-Pohst-Martinet's Theorem [19].

Theorem 2.1. Let $K$ be a number field of degree $n$ with discriminant $d_{K}$. Then there exists an element $\alpha \in \mathcal{O}_{K} \backslash \mathbb{Z}$ which satisfies the following conditions:

(1) $0 \leq \operatorname{Tr}(\alpha) \leq\left\lfloor\frac{n}{2}\right\rfloor$;

(2) $T_{2}(\alpha) \leq \frac{\operatorname{Tr}(\alpha)^{2}}{n}+\gamma_{n-1}\left(\frac{\left|d_{K}\right|}{n}\right)^{1 /(n-1)}=: U_{2}$

where $\gamma_{n-1}$ is Hermite's constant in dimension $n-1$. 
The element $\alpha$ is called an HPM-element for $K$ : for such an algebraic integer, the previous theorem allows us to compute an upper bound for its trace and its second Newton sum $S_{2}$. These data, together with the absolute value of the norm $N:=|\mathrm{Nm}(\alpha)|$, are enough for giving upper bounds to every Newton sum, thanks to Pohst's result [28].

Theorem 2.2. Given $K, U_{2}$ and $\alpha$ as in Theorem 2.1, given $N \in \mathbb{N}$ such that $N \leq\left(U_{2} / n\right)^{n / 2}$, then for every $k \in \mathbb{Z} \backslash\{0,2\}$ we have an inequality

$$
\left|S_{k}(\alpha)\right| \leq U_{k}
$$

where $U_{k}$ is an explicit number depending on $n, r_{1}$ and $U_{2}$.

Our goal is to test the polynomials generated by a choice of the coefficients which derives from the values of the Newton sums $S_{k}$ (with $2 \leq k \leq n)$ ranging in the intervals $\left[-U_{k}, U_{k}\right]$ and satisfying the recursive relations (2.1). In order to do so, we need to choose an upper bound for $\left|d_{K}\right|$.

Remark 2.3. The condition $N \leq\left(U_{2} / n\right)^{n / 2}$ is set in order to respect the inequality between geometric and arithmetic means: in fact,

$$
N^{2}=\prod_{i=1}^{n}\left|\alpha_{i}\right|^{2} \leq\left(\frac{\sum_{i=1}^{n}\left|\alpha_{i}\right|^{2}}{n}\right)^{n}=\left(\frac{U_{2}}{n}\right)^{n} .
$$

We recall now an inequality, proved by Poitou [30], which gives a lower bound for the discriminants of number fields with fixed degree $n$ and signature $\left(r_{1}, r_{2}\right)$.

Theorem 2.4. Let $K$ be a number field of degree $n$, signature $\left(r_{1}, r_{2}\right)$ and discriminant $d_{K}$. Let $f(x)$ be the function

$$
f(x):=\left(\frac{3}{x^{3}}(\sin x-x \cos x)\right)^{2} .
$$

Then, for every $y>0$, one has

$$
\begin{aligned}
\frac{1}{n} \log \left|d_{K}\right| \geq \gamma+ & \log 4 \pi-L_{1}(y)-\frac{12 \pi}{5 n \sqrt{y}} \\
& +\frac{4}{n} \sum_{\mathfrak{p} \subset \mathcal{O}_{K}} \sum_{m=1}^{\infty} \frac{\log \mathrm{Nm}(\mathfrak{p})}{1+(\mathrm{Nm}(\mathfrak{p}))^{m}} f(m \sqrt{y} \log \mathrm{Nm}(\mathfrak{p}))
\end{aligned}
$$

where $\gamma$ is Euler's constant, the sum runs over the non-zero prime ideals of $\mathcal{O}_{K}, \mathrm{Nm}(\mathfrak{p})$ is the absolute norm of the prime $\mathfrak{p}$ and

$$
L_{1}(y):=\sum_{k=1}^{\infty} \frac{1}{2 k-1} L\left(\frac{y}{(2 k-1)^{2}}\right)+\frac{r_{1}}{n} \sum_{k=1}^{\infty}(-1)^{k-1} L\left(\frac{y}{k^{2}}\right)
$$


where

$L(y):=-\frac{3}{20 y^{2}}+\frac{33}{10 y}+2+\left(\frac{3}{80 y^{3}}+\frac{3}{4 y^{2}}\right)\left(\log (1+4 y)-\frac{1}{\sqrt{y}} \arctan (2 \sqrt{y})\right)$.

Assume that we are able to guarantee that a prime ideal $\mathfrak{p}$ with a fixed norm is contained in $\mathcal{O}_{K}$ : then the estimate $(2.2)$ can be reduced to an explicit inequality of the form

$$
\frac{1}{n} \log \left|d_{K}\right| \geq B_{y}\left(r_{1}, r_{2}\right)+\frac{4}{n} F_{y}(\mathrm{Nm}(\mathfrak{p}))
$$

where

$B_{y}\left(r_{1}, r_{2}\right):=\gamma+\log 4 \pi-L_{1}(y)-\frac{12 \pi}{5 n \sqrt{y}}, \quad F_{y}(q):=\sum_{m=1}^{\infty} \frac{\log q}{1+q^{m}} f(m \sqrt{y} \log q)$.

The term in inequality (2.3) which depends explicitly on $\mathfrak{p}$ is called local correction given by an ideal of norm $\mathrm{Nm}(\mathfrak{p})$. Looking for the value of $y$ which maximizes the right hand side of (2.3), we obtain a lower bound $C\left(r_{1}, r_{2}, \operatorname{Nm}(\mathfrak{p})\right)$ called lower bound for the discriminant with the local correction given by an ideal of norm $\mathrm{Nm}(\mathfrak{p})$. It is important to underline that different signatures and norms may require different values of $y$ for a proper optimization.

Selmane [33] computed the values of local corrections and of the corresponding lower bounds for several signatures and prime ideals; these values are reported in the following tables, which present lower bounds for $\left|d_{K}\right|$ for fields of degree 8 and 9 in every signature. The first line in each table consists of absolute lower bounds obtained by Diaz y Diaz [10] by optimization of $B_{y}$ only, without any local correction; the remaining lines present instead lower bounds with local corrections given by prime ideals $\mathfrak{p}$ with $\operatorname{norm} \operatorname{Nm}(\mathfrak{p}) \leq 7$.

TABLE 2.1. Lower bounds $C\left(r_{1}, r_{2}, \operatorname{Nm}(\mathfrak{p})\right)$ with local corrections for fields of degree 8

\begin{tabular}{l|c|c|c|c|c|}
$\left(r_{1}, r_{2}\right)$ & $(0,4)$ & $(2,3)$ & $(4,2)$ & $(6,1)$ & $(8,0)$ \\
\hline No local corrections & 1052302 & 3403708 & 11660853 & 42071532 & 158960873 \\
\hline $\operatorname{Nm}(\mathfrak{p})=2$ & 3379343 & 11725962 & 42765027 & 163060410 & 646844001 \\
\hline $\mathrm{Nm}(\mathfrak{p})=3$ & 2403757 & 8336752 & 30393063 & 115852707 & 459467465 \\
\hline $\mathrm{Nm}(\mathfrak{p})=4$ & 1930702 & 6688609 & 24363884 & 92810084 & 367892401 \\
\hline $\mathrm{Nm}(\mathfrak{p})=5$ & 1656110 & 5726300 & 20829049 & 79259702 & 313918560 \\
\hline $\mathrm{Nm}(\mathfrak{p})=7$ & 1362891 & 4682934 & 16957023 & 64309249 & 254052210
\end{tabular}

Lower bounds with local corrections provide the following arithmetic consequences: if $K$ has signature $\left(r_{1}, r_{2}\right)$ and $\left|d_{K}\right|<C\left(r_{1}, r_{2}, \operatorname{Nm}(\mathfrak{p})\right)$, then $\mathcal{O}_{K}$ does not admit any prime ideal with norm less or equal than $\operatorname{Nm}(\mathfrak{p})$. 
TABLE 2.2. Lower bounds $C\left(r_{1}, r_{2}, \mathrm{Nm}(\mathfrak{p})\right)$ with local corrections for fields of degree 9

\begin{tabular}{l|c|c|c|c|c|}
$\left(r_{1}, r_{2}\right)$ & $(1,4)$ & $(3,3)$ & $(5,2)$ & $(7,1)$ & $(9,0)$ \\
\hline No local corrections & 23007468 & 80499454 & 295584269 & 1133345241 & 4516673524 \\
\hline $\operatorname{Nm}(\mathfrak{p})=2$ & 81295493 & 301476699 & 1165734091 & 4679379812 & 19422150186 \\
\hline $\operatorname{Nm}(\mathfrak{p})=3$ & 57789556 & 214235371 & 828172359 & 3323651196 & 13792634200 \\
\hline $\operatorname{Nm}(\mathfrak{p})=4$ & 46348899 & 171694276 & 663330644 & 2660853331 & 11037921283 \\
\hline $\operatorname{Nm}(\mathfrak{p})=5$ & 39657561 & 146723910 & 566314434 & 2269968332 & 9410709985 \\
\hline $\operatorname{Nm}(\mathfrak{p})=7$ & 32371189 & 119294181 & 459066389 & 1835807996 & 7596751280
\end{tabular}

This fact has consequences on the defining polynomials of the field: assume that $\left|d_{K}\right|$ is less than $C\left(r_{1}, r_{2}, \operatorname{Nm}(\mathfrak{p})\right)$. If $p(x)$ is a defining polynomial of $K$ and $\alpha \in K$ is a root of $p(x)$, then we know that $|p(n)|=$ $\operatorname{Nm}\left((\alpha-n) \mathcal{O}_{K}\right)$ for every $n \in \mathbb{Z}$, and so $p(n)$ must not be an exact multiple of every $m \in\{2, \ldots, \operatorname{Nm}(\mathfrak{p})\}$, where a number $a$ is said to be an exact multiple of $b$ if $b$ divides $a$ and $a / b$ is not divisible by $b$. If $m=l$ is prime, the required condition becomes $v_{l}(p(n)) \neq 1$, where $v_{l}$ is the $l$-adic discrete valuation.

\section{The Algorithmic Procedure}

We want to detect all the number fields $K$ with degree $n$, signature $\left(r_{1}, r_{2}\right)$ and $\left|d_{K}\right| \leq C\left(r_{1}, r_{2}, 5\right)$, where $C\left(r_{1}, r_{2}, 5\right)$ is the lower bound for the signature $\left(r_{1}, r_{2}\right)$ with local correction given by a prime ideal of norm 5 . We accomplish this by constructing all the polynomials of degree $n$ having integer coefficients bounded by the values $U_{m}$ obtained from Theorems 2.1 and 2.2 , setting $C\left(r_{1}, r_{2}, 5\right)$ as upper bound of $\left|d_{K}\right|$. Thanks to this construction, it is clear that we are dealing with defining polynomials of HPM-elements.

The polynomials are generated ranging the values for the Newton sums $S_{m}$ in the intervals $\left[-U_{m}, U_{m}\right]$; from these values we create the coefficients of the polynomials with the help of the recursive relations (2.1) and of further conditions derived from the arithmetic nature of the problem, like the fact that any evaluation of the polynomial cannot be an exact multiple of $2,3,4$ or 5 .

Remark 3.1. As stated above, the procedure assumes that we are looking for defining polynomials of HPM-elements. There is a problem, however: unless the number field $K$ is primitive, i.e. without subfields which are not $\mathbb{Q}$ and $K$, nothing assures us that the defining polynomial of an HPM element $\alpha \in K$ has degree exactly equal to $n$. In fact, $\alpha$ could be contained in a proper subfield of $K$. 
So this procedure gives a complete classification only for primitive fields, which for composite degrees is still a proper subset of the considered family (though being actually a very large subset).

Fortunately, a relative version of Hunter-Pohst-Martinet's Theorem [19] allowed to get a complete classification of non-primitive fields up to larger upper bounds for $\left|d_{K}\right|$, and specifically in the following cases:

- [8] and [32] give a complete classification of non-primitive fields of degree 8 with signature $(2,3),(4,2)$ and $(6,1)$ and $\left|d_{K}\right| \leq 6688609$, 24363884 and 92810082 respectively;

- $[15]$ gives a classification of non-primitive number fields of degree 9 with $\left|d_{K}\right| \leq 5 \cdot 10^{7}, 4 \cdot 10^{9}, 5 \cdot 10^{9}, 7 \cdot 10^{9}, 6,3 \cdot 10^{10}$ for the signatures $(1,4),(3,3),(5,2),(7,1),(9,0)$ respectively.

Thus in our procedure we can restrict ourselves to primitive fields.

For what concerns the algorithmic procedure, we underline that is in fact the same we used in order to classify number fields with signature $(2,3)$, so that we will not give all the details here, but we will just refer to what is presented in [3, Section 4]. In fact, we obtained Theorem 1.1 by following the instructions of the previous algorithm from Step 0 to Step 4.

There are nonetheless some differences which must be remarked: first of all, one should replace the previous upper bound 5762300 with $C\left(r_{1}, r_{2}, 5\right)$, the lower bound with local correction at ideals of norm 5; moreover, every feature in the algorithm related to the previously chosen degree $n=8$, like the amount of nested loops or the checks done in Step 3, can be easily generalized for an arbitrary degree $n$.

Next, there are some additional tests that can be made already in Step 1: the polynomial $p(x)$ is kept if and only if it is constructed by Newton sums satisfying the followings restraints.

$$
\begin{aligned}
& \text { If } a_{1}=0, \text { then } S_{3} \geq 0, \\
& S_{2} \geq-U_{2}+\frac{2}{n} a_{1}^{2}, \\
& \left|S_{3}\right| \leq\left(\frac{S_{2}+U_{2}}{2}\left(S_{4}+2\left(U_{2}-S_{2}\right)^{2}\right)\right)^{1 / 2}, \\
& S_{4} \geq-2\left(U_{2}-S_{2}\right)^{2} .
\end{aligned}
$$

The first two inequalities are proved in Cohen's book [7, Chapter 9]. Inequality (3.1) is proved by means of Cauchy-Schwartz inequality. The fourth inequality is a trivial necessary condition for the validity of the third one.

Finally, Step 5 of the previous version is now put into Step 3, so that a candidate polynomial $p(x)$ for defining a desired number field should satisfy, together with the conditions described in Step 3, the following properties. 
- $p(x)$ must be an irreducible polynomial.

- The field generated by $p(x)$ must not have prime ideals of norm less or equal than 5 . This can be verified in an algorithmic way (as we explain in the next section). Moreover, the signature of $p(x)$ must be equal to $\left(r_{1}, r_{2}\right)$.

- Given an integer $m$, define coredisc $(m)$ as the discriminant of the number field $\mathbb{Q}(\sqrt{m})$. Then we require $|\operatorname{coredisc}(\operatorname{disc}(p(x)))|<$ $C\left(r_{1}, r_{2}, 5\right)$ : this is justified by the fact that, if $p(x)$ is an irreducible integer polynomial, then $|\operatorname{coredisc}(\operatorname{disc}(p))| \leq \mid \operatorname{disc}(\mathbb{Q}[x] /(p(x)) \mid$.

Once we have followed the instructions from Step 0 to Step 4, comprehensive of the above modifications, one just needs to replace the previous Step 5 with the following Step $5^{\prime}$.

Step $\mathbf{5}^{\prime}$. We repeat the previous steps for every value of $a_{1}$ between 0 and $n / 2$ and for every value of $a_{n}$ which satisfies $\left|a_{n}\right| \leq\left(U_{2} / n\right)^{n / 2}$ and is not an exact multiple of 2, 3, 4 or 5 . We are left with a list of polynomials among which we select the ones generating a number field $K$ with signature $\left(r_{1}, r_{2}\right)$ and $\left|d_{K}\right| \leq C\left(r_{1}, r_{2}, 5\right)$.

The gathered fields are finally classified up to isomorphism and put in increasing order with respect to their absolute discriminant.

\section{Remarks on the implementation and the results}

The theoretical ideas on which our procedure is based and the several steps composing the algorithm are very similar to what has been introduced in [3], with only few differences in some of the conditions put during the tests (like the check on the size of the coredisc). The main novelty which allowed us to obtain complete tables for further signatures is the different implementation, written only in PARI/GP, which gave the following consequences and facts:

- As previously mentioned, the polynomials created during the process are tested by verifying that the ideals generated by the corresponding number fields do not have norm less or equal than 5 . The implementation of this process has been achieved thanks to the ZpX-primedec() function, written by Karim Belabas on purpose: the function is theoretically based upon the work by Ford, Pauli and Roblot ([16, Section 6]) which use the Round 4 Algorithm in order to recover the factorization of a prime ideal from the $l$-adic factorization of a minimal polynomial of the field.

For what concerns its efficiency, this function is an order of magnitude faster than the partial factorization given by nfinit() and faster than the usual decomposition function idealprimedec(): 
moreover, it is even faster whenever the valuation at $l$ of the in$\operatorname{dex}\left[\mathcal{O}_{K}: \mathbb{Z}[X] /(p)\right]$ is small.

- The final check on the polynomials, suggested by Bill Allombert, concerns the size of $\operatorname{core}(\operatorname{disc}(p(x)))$ : this test was added only some month after the signatures in degree 8 were solved. However, it allows to exclude many polynomials, because several candidate polynomials $p(x)$ have in fact core discriminants with very big size, which would force the number field discriminant to be way over the desired upper bound.

The number of polynomials surviving this last condition is very small, at most 100 in our examples, and for these one can directly compute the number field discriminant.

- The times of computation vary considerably and range from few hours (for signatures $(2,3)$ and $(4,2)$ ), few days (signatures $(6,1)$ and $(1,4))$, up to some months (signature $(3,3)$ ).

- The tables presenting all the detected number fields can be found as PARI/GP files at the website [2], together with the programs written by the author, the collection of polynomials found as result of the iterations and the overview on computation times.

Finally, we present some remarks concerning the results described in Theorem 1.1.

- Every field in our lists is uniquely characterized by its signature and the value of its discriminant, with exceptions given only by two fields with signature $(3,3)$ and same discriminant equal to -142989047 . These fields are given by the defining polynomials $x^{9}-4 x^{7}-4 x^{6}+$ $2 x^{5}+5 x^{4}+6 x^{3}+8 x^{2}+4 x+1$ and $x^{9}-6 x^{7}-9 x^{6}-2 x^{5}+21 x^{4}+$ $35 x^{3}+23 x^{2}+7 x+1$.

- Every field of degree 8 and every field with signature $(1,4)$ contained in our lists was already known: in fact, they are all gathered into the Klüners-Malle database of number fields [18], although many of them are missing from the LMFDB database [37]. Our work allows to say that these are the only number fields with the corresponding signatures with discriminant less than the chosen upper bound.

- Concerning the fields of degree 9 and signature $(3,3)$, our procedure showed that there exist 116 such fields with $\left|d_{K}\right| \leq 146723910$, while the Klüners-Malle database only contains 62 fields of this kind. Considering the additional 54 fields, we see that 52 of them have discriminant which match with Denis Simon's table of small polynomial discriminants [35]. The two remaining fields satisfy instead the following properties: one of them is the field of discriminant -142989047 which is not isomorphic to the one given by the 
polynomial in Simon's list mentioned above; the other one has discriminant equal to -129079703 , which is a value not contained in Simon's lists for polynomials of degree 9 with 3 real roots, thus providing a number field and a discriminant value which were not previously known.

- Every field in the list has trivial class group, and every primitive field has Galois group $G$ of the Galois closure equal to either $S_{8}$ or $S_{9}$, depending on the degree. More in detail, we have:

- 27 primitive fields out of 41 fields with signature $(4,2)$ (65.8\% ca.).

- 4 primitive fields out of 8 fields with signature $(6,1)(50 \%)$.

- 63 primitive fields out of 67 fields with signature $(1,4)(94 \%$ ca.).

- 112 primitive fields out of 116 fields in signature $(3,3)$ (96.5\% ca.). Furthermore, Table 4.1 provides the minimum values of $\left|d_{K}\right|$ for a field $K$ with signature $\left(r_{1}, r_{2}\right)$ and with Galois group $G$ as reported.

TABle 4.1. Minimum discriminants for specific Galois groups

\begin{tabular}{|c|c|c|c|}
$n$ & $\left(r_{1}, r_{2}\right)$ & $G$ & minimum $\left|d_{K}\right|$ \\
\hline 8 & $(4,2)$ & $S_{8}$ & 15908237 \\
\hline 8 & $(6,1)$ & $S_{8}$ & 65106259 \\
\hline 9 & $(1,4)$ & $S_{9}$ & 29510281 \\
\hline 9 & $(3,3)$ & $S_{9}$ & 109880167 \\
\hline
\end{tabular}

- Although the algorithm classifies only primitive fields, every nonprimitive field with $\left|d_{K}\right| \leq C\left(n, r_{1}, 5\right)$ was displayed as output.

- The groups in [2] are presented according to the LMFDB notation: every group is denoted by $n \mathrm{~T} q$, where $n$ is the degree of the corresponding field and $q$ is the label of the group as transitive subgroup of $S_{n}$ : the choice of the label is based upon Hulpke's algorithm for the classification of transitive subgroups of $S_{n}$ [17]. For standard groups, like the dihedral group $D_{n}$ or the symmetric group $S_{n}$, the classical name of the group is written together with the LMFDB label.

\section{References}

[1] S. Astudillo, F. Diaz y Diaz \& E. Friedman, "Sharp lower bounds for regulators of small-degree number fields", J. Number Theory 167 (2016), p. 232-258.

[2] F. Battistoni, "Tables of Number Fields", available at http://www.mat.unimi.it/users/ battistoni/index.html.

[3] - "The minimum discriminant of number fields of degree 8 and signature $(2,3)$ ", $J$. Number Theory 198 (2019), p. 386-395.

[4] K. Belabas, "A fast algorithm to compute cubic fields", Math. Comput. 66 (1997), no. 219, p. $1213-1237$.

[5] A.-M. Bergé, J. Martinet \& M. Olivier, "The computation of sextic fields with a quadratic subfield", Math. Comput. 54 (1990), no. 190, p. 869-884. 
[6] J. Buchmann, D. Ford \& M. Pohst, "Enumeration of quartic fields of small discriminant", Math. Comput. 61 (1993), no. 204, p. 873-879.

[7] H. Cohen, Advanced topics in computational number theory, Graduate Texts in Mathematics, vol. 193, Springer, 2000.

[8] H. Cohen, F. Diaz y Diaz \& M. Olivier, "Tables of octic fields with a quartic subfield", Math. Comput. 68 (1999), no. 228, p. 1701-1716.

[9] H. Davenport \& H. Heilbronn, "On the density of discriminants of cubic fields. II", Proc. R. Soc. Lond., Ser. A 322 (1971), p. 405-420.

[10] F. Diaz y Diaz, Tables minorant la racine $n$-ième du discriminant d'un corps de degré $n$, Publications Mathématiques d'Orsay, vol. 6, 1980.

[11] - "Valeurs minima du discriminant des corps de degré 7 ayant une seule place réelle", C. R. Math. Acad. Sci. Paris 296 (1983), p. 137-139.

[12] - "Valeurs minima du discriminant pour certains types de corps de degré 7", Ann. Inst. Fourier 34 (1984), no. 3, p. 29-38.

[13] - "Petits discriminants des corps de nombres totalement imaginaires de degré 8", $J$. Number Theory 25 (1987), p. 34-52.

[14] - "Discriminant minimal et petits discriminants des corps de nombres de degré 7 avec cinq places réelles", J. Lond. Math. Soc. 38 (1988), no. 1, p. 33-46.

[15] F. Diaz y Diaz \& M. Olivier, "Imprimitive ninth-degree number fields with small discriminants", Math. Comput. 64 (1995), no. 209, p. 305-321.

[16] D. Ford, S. PAuli \& X.-F. Roblot, "A fast algorithm for polynomial factorization over $\mathbb{Q}_{p}$ ", J. Théor. Nombres Bordeaux 14 (2002), no. 1, p. 151-169.

[17] A. Hulpke, "Constructing transitive permutation groups", J. Symb. Comput. 39 (2005), no. 1, p. 1-30.

[18] J. KlüNers \& G. MAlle, "A database for number fields", available at http://galoisdb. math.upb.de/home.

[19] J. Martinet, "Méthodes géométriques dans la recherche des petits discriminants", in Séminaire de théorie des nombres, Progress in Mathematics, vol. 59, Birkhäuser, 1983, p. 1983-84.

[20] A. M. OdLyzko, "Bounds for discriminants and related estimates for class numbers, regulators and zeros of zeta functions: a survey of recent results", Sémin. Théor. Nombres Bordx., Sér. II 2 (1990), no. 1, p. 119-141.

[21] M. Olivier, "Corps sextiques contenant un corps quadratique. I", Sémin. Théor. Nombres Bordx., Sér. II 1 (1989), no. 1, p. 205-250.

[22] - "Corps sextiques contenant un corps quadratique. II", Sémin. Théor. Nombres Bordx., Sér. II 2 (1990), no. 1, p. 49-102.

[23] — "Corps sextiques primitifs", Ann. Inst. Fourier 40 (1990), no. 4, p. 757-767.

[24] - "Corps sextiques contenant un corps cubique. III", Sémin. Théor. Nombres Bordx., Sér. II 3 (1991), no. 1, p. 201-245.

[25] - "Corps sextiques primitifs. IV", Sémin. Théor. Nombres Bordx., Sér. II 3 (1991), no. 2, p. 381-404.

[26] - "The computation of sextic fields with a cubic subfield and no quadratic subfield", Math. Comput. 58 (1992), no. 197, p. 419-432.

[27] M. Pohst, "The minimum discriminant of seventh degree totally real algebraic number fields", in Number theory and algebra, Academic Press Inc., 1977, p. 235-240.

[28] - "On the computation of number fields of small discriminants including the minimum discriminants of sixth degree fields", J. Number Theory 14 (1982), p. 99-117.

[29] M. Pohst, J. Martinet \& F. Diaz y Diaz, "The minimum discriminant of totally real octic fields", J. Number Theory 36 (1990), no. 2, p. 145-159.

[30] G. Poitou, "Sur les petits discriminants", in Séminaire Delange-Pisot-Poitou, 18e année: (1976/77), Théorie des nombres, Fasc. 1, Secrétariat Mathématique, 1976.

[31] A. Schwarz, M. Pohst \& F. Diaz y Diaz, "A table of quintic number fields", Math. Comput. 63 (1994), no. 207, p. 361-376.

[32] S. Selmane, "Non-primitive number fields of degree eight and of signature $(2,3),(4,2)$ and $(6,1)$ with small discriminant", Math. Comput. 68 (1999), no. 225, p. 333-344. 
[33] - "Odlyzko-Poitou-Serre lower bounds for discriminants for some number fields", Maghreb Math. Rev. 8 (1999), p. 151-162.

[34] J.-P. Serre, "Minorations de discriminants", in Jean-Pierre Serre, collected papers. Vol. 3, vol. 3,1975 , note of october 1975 , p. $240-243$.

[35] D. Simon, "Petits discriminants de polynomes irréductibles", available at https://simond. users.lmno.cnrs.fr/maths/TableSmallDisc.html.

[36] K. TAKeuchi, "Totally real algebraic number fields of degree 9 with small discriminant", Saitama Math. J. 17 (2000), p. 63-85.

[37] The LMFDB Collaboration, "The L-functions and Modular Forms Database", 2013, http://www. lmfdb.org.

[38] The PARI Group, "PARI/GP version 2.11.0", 2018, available from http://pari.math. u-bordeaux.fr/.

Francesco BATtistoni

Dipartimento di Matematica

Università di Milano

via Saldini 50

20133 Milano, Italy

E-mail: francesco.battistoni@unimi.it

$U R L:$ http://www.mat.unimi.it/users/battistoni 